\title{
Stable Metabolic Disease
}

National Cancer Institute

\section{Source}

National Cancer Institute. Stable Metabolic Disease. NCI Thesaurus. Code C123616.

The absence of change or mild changes of metabolic tumor activity in all relevant lesions based on a pre-defined threshold. 